\title{
Integrative genomic analyses on Ikaros and its expression related to solid cancer prognosis
}

\author{
LIMING YANG ${ }^{1}$, YUMING LUO $^{1}$ and JIFU WEI ${ }^{2}$ \\ ${ }^{1}$ School of Life Sciences, Huaiyin Normal University, 111 Changjiang West Road, Huai'an 223300; \\ ${ }^{2}$ Clinical Research Center, the First Affiliated Hospital of Nanjing Medical University, \\ 300 Guangzhou Road, Nanjing 210029, P.R. China
}

Received March 10, 2010; Accepted May 3, 2010

DOI: $10.3892 /$ or_00000894

\begin{abstract}
Ikaros is a member of the Kruppel family of zinc finger DNA-binding proteins. The Ikaros protein contains two separate regions of zinc-finger domains: 4 DNA-binding zinc fingers near the $\mathrm{N}$-terminus and 2 zinc fingers for proteinprotein interactions near the $\mathrm{C}$-terminus. Here, we identified the Ikaros gene from 14 vertebrate genomes and found Ikaros existed in all kinds of vertebrate including fish, amphibians, birds and mammals. Moreover, except rat and Xenopus tropicalis Ikaros proteins, which lack the first $\mathrm{C} 2 \mathrm{H} 2$-type 1 Zinc finger region, all identified Ikaros proteins contain six $\mathrm{C} 2 \mathrm{H} 2$-type 1 Zinc finger regions. We found human Ikaros gene showed a predominant expression in the liver, lymph node, thymus, intestine, lung, mammary gland, bone marrow, brain, heart, placenta and prostate. Moreover, four available SNPs disrupted an existing exonic splicing enhancer were identified in Ikaros. Besides the reported acute lymphoblastic leukemia (ALL), the expression of Ikaros was related to the prognosis of 13 cases of cancers including blood cancers, breast, lung, ovarian and skin cancer. Moreover, the relationship between the expression of Ikaros and prognosis varied in different cancers, even in the same cancer from different database. Two tumor-related transcriptional factor (c-Fos and Elk-1) binding sites were identified within the $1.5-\mathrm{kb}$ regions upstream of the transcriptional start site of human Ikaros, which may be involved in the effect of Ikaros in tumors.
\end{abstract}

Correspondence to: Dr Liming Yang, School of Life Sciences, Huaiyin Normal University, 111 Changjiang West Road, Huai'an 223300, P.R. China

E-mail: yanglm@hytc.edu.cn or

Dr Jifu Wei, Clinical Research Center and the First Affiliated Hospital of Nanjing Medical University, 300 Guangzhou Road, Nanjing 210029, P.R. China

E-mail:weijifu@hotmail.com

Key words: Ikaros, comparative genomics, comparative proteomics, cancer, prognosis, meta-analysis

\section{Introduction}

The Ikaros (IKZF1, Lyf-1) is a member of the Kruppel family of zinc finger DNA-binding proteins 1 (1). The Ikaros protein contains two separate regions of zinc-finger domains: 4 DNA-binding zinc fingers near the N-terminus and 2 zinc fingers for protein-protein interactions near the $\mathrm{C}$-terminus. The human Ikaros gene, located at 7p12, contains seven exons and gives rise to at least eight isoforms by alternative splicing (2). All isoforms share a common C-terminal domain that contains a transcriptional activation domain and two zinc finger motifs required for hetero- or homodimerization and for interactions with other proteins, but these isoforms differ in the number of $\mathrm{N}$-terminal zinc finger motifs. Long isoforms (Ik1 to 3) have at least three zinc fingers which are capable of binding DNA and considered to be functional. Short isoforms (Ik4 to 8) lack two or more zinc-finger domains, so they cannot bind DNA and impair the function of Ikaros proteins in a dominant-negative manner (3-6).

Ikaros is a transcription factor, which plays an important role in controlling hematopoietic, particularly lymphoid cell differentiation, proliferation and function by binding upstream regulatory regions of target genes and aiding in their recruitment to pericentromeric heterochromatin (PC-HC) (7). This process leads to either activation or repression of transcription of these target genes by elaborate splicing regulation of Ikaros transcripts (3-6). Accordingly, abnormalities in splicing regulation of Ikaros would lead to significant pathological manifestations $(3,4,8,9)$. Mice that are heterozygous for a germline mutation that results in a loss of critical DNA-binding zinc fingers of Ikaros develop a very aggressive form of lymphoblastic leukemia, suggesting that Ikaros has an important tumor suppressor function $(10,11)$. Approximately $30 \%$ of pediatric B-cell acute lymphoblastic leukemia (ALL) cases showed genetic inactivation of Ikaros due to deletion or mutations (12-14). High-level expression of dominant-negative isoforms of Ikaros with abnormal subcellular compartmentalization patterns were also found in T-cell ALL (15) and pituitary tumors (16). However, whether Ikaros is involved in other tumors formations, especially solid tumors, is still unknown.

In the present study, we identified Ikaros genes from human, chimpanzee, macaque, orangutan, dog, cow, horse, mouse, rat, opossum, chicken, Xenopus tropicalis, zebrafish, 
and fugu by comparative genomic analyses. Conserved transcription factor-binding sites within promoter regions of human Ikaros genes were then searched. The expression data, functional relevant single nucleotide polymorphisms (SNPs) and comparative proteomic analyses were conducted. Furthermore, meta-analysis of the prognostic value of Ikaros genes in various cancers was also performed.

\section{Materials and methods}

Identification of novel Ikaros genes in vertebrate genomes and integrative genomic analyses. Ikaros genes were searched for in the genome sequences of human (Homo sapiens), chimpanzee (Pan troglodytes), macaque (Macaca mulatta), orangutan (Pongo pygmaeus), dog (Canis familiaris), cow (Bos taurus), horse (Equus caballus), mouse (Mus musculus), rat (Rattus norvegicus), opossum (Monodelphis domestica), chicken (Gallus gallus), Xenopus tropicalis, zebrafish (Danio rerio), and fugu (Takifugu rubripes) by the method described before using human Ikaros gene (NM_006060) as queries. The assemblies used were human NCBI 36, chimpanzee CHIMP2.1, macaque MMUL 1.0, orangutan PPYG2, dog Canfam 2.0, cow Btau_4.0, horse Equ Cab 2, mouse NCBI m37, rat RGSC 3.4, opossum monDom5, chicken WASHUC2, X. tropicalis JGI 4.1, zebrafish Zv8 and fugu FUGU 4.0. The identified putative Ikaros genes were BLASTed against the database number of GenBank to confirm that the best hits were Ikaros genes. Conserved transcription factor-binding sites within promoter region of human Ikaros gene were then searched for based on the Patch program (http://www.gene-regulation.com) as well as manual inspection as previously described (17-28).

Comparative proteomic analyses of Ikaros proteins. The amino acid sequences of Ikaros were deduced from the identified Ikaros genes and aligned using Clustal X 1.8 software (29). The phylogenetic tree of Ikaros was obtained by using ML (maximum likelihood) (PHYML v2.4.4) (30 and NJ (neighbor-joining) (MEGA 3.0) (31) methods, and the reliability of the tree was evaluated by the bootstrap method with 1,000 replications. The program Codeml implemented in the PAML 3.14 b software package was used to investigate whether Ikaros proteins are under positive selection (32). Six models of codon substitution, M0 (one-ratio), M1a (NearlyNeutral), M2a (PositiveSelection), M3 (discrete), M7 $(ß)$, and M8 ( $ß$ and $\omega$ ) were used in the analysis (33).

Functional relevant SNP evaluation of human Ikaros gene. Functional relevant SNPs (single nucleotide polymorphisms) of human Ikaros gene were identified as previously described $(34,35)$. The SNPs were extracted from Ensembl (http:// www.ensembl.org) and NCBI's SNPdb (http://www.ncbi. nlm.nih.gov). The SNPs that could disrupt ESE/ESS (exonic splicing enhancer/exonic splicing silencer) motifs and cause missense mutation were also identified.

In silico expression analyses of human Ikaros gene. Expressed sequence tags (ESTs) derived from human Ikaros were searched for using the BLAST programs as previously described (15-19). Human Ikaros gene (NM_006060) was used as query sequences for the BLAST programs. The expression profiles for normal human tissues were obtained from GeneAnnot (36) and ArrayExpress (37). Northern analysis of NCBI's uniGene dataset was also extracted $(34,35)$.

Meta-analysis of the prognostic value of Ikaros gene in cancer. A database named 'PrognoScan' has been developed (36). This is: i) a large collection of publicly available cancer microarray datasets with clinical annotation, as well as ii) a tool for assessing the biological relationship between gene expression and prognosis. PrognoScan employs the minimum P-value approach for grouping patients for survival analysis, and it provides a powerful platform for evaluating potential tumor markers and therapeutic targets and is publicly accessible at http://gibk21.bse.kyutech.ac.jp/PrognoScan/ index.html. Human Ikaros (IKZF1) gene was inputted as queries and the data were collected for analysis.

\section{Results}

Comparative proteomics of Ikaros proteins identified in vertebrate genomes. Ikaros genes were identified in the genome sequences of human, chimpanzee, macaque, orangutan, dog, cow, horse, mouse, rat, opossum, chicken, Xenopus tropicalis, zebrafish and fugu. Their amino acid sequences are shown in alignment format in Fig. 1. Except rat and Xenopus tropicalis Ikaros proteins, which lack the first $\mathrm{C} 2 \mathrm{H} 2$-type 1 Zinc finger region, all identified Ikaros proteins containing six $\mathrm{C} 2 \mathrm{H} 2$-type 1 Zinc finger regions (Fig. 1). Refined phylogenetic trees using the identified Ikaros amino acid sequences by ML and NJ methods were almost the same (Fig. 2). It seemed that primate Ikaros proteins clustered into one group, different from other Ikaros proteins. We were unable to identify any site under positive selection with any of the six models in Ikaros proteins. Instead, the Ikaros proteins were under purifying selection (data not shown).

Expression profile of human Ikaros gene. By EST sequence search, human Ikaros gene was expressed in parathyroid, liver, lymph node, stomach, uterus, vascular, thymus, muscle, pharynx, intestine, ovary, thyroid, lung, mammary gland, blood, bone, bone marrow, brain, spleen, heart, placenta, tonsil, prostate, and connective tissue. The investigation of available microarray experiments and 'virtual northern blot' showed a predominant expression of Ikaros in the liver, lymph node, thymus, intestine, lung, mammary gland, bone marrow, brain, heart, placenta, and prostate tissue. When searched in PrognoScan database, human Ikaros was also found expressed in bladde, blood, breast cancer, gliomas, colorectal, head and neck, ovarian, lung and skin cancer tissues.

Comparative genomics on the human Ikaros gene. Transcription factor-binding sites within the 5'-region of Ikaros gene were identified (Fig. 3). The c-Fos, E-26-like protein 1 (Elk-1), GATA-1 and Nk6 homeobox gene-B (NKX6-B) binding sites were identified within the $1.5-\mathrm{kb}$ regions upstream of the transcriptional start site of human Ikaros gene. Functional relevant SNP evaluation showed that 130 


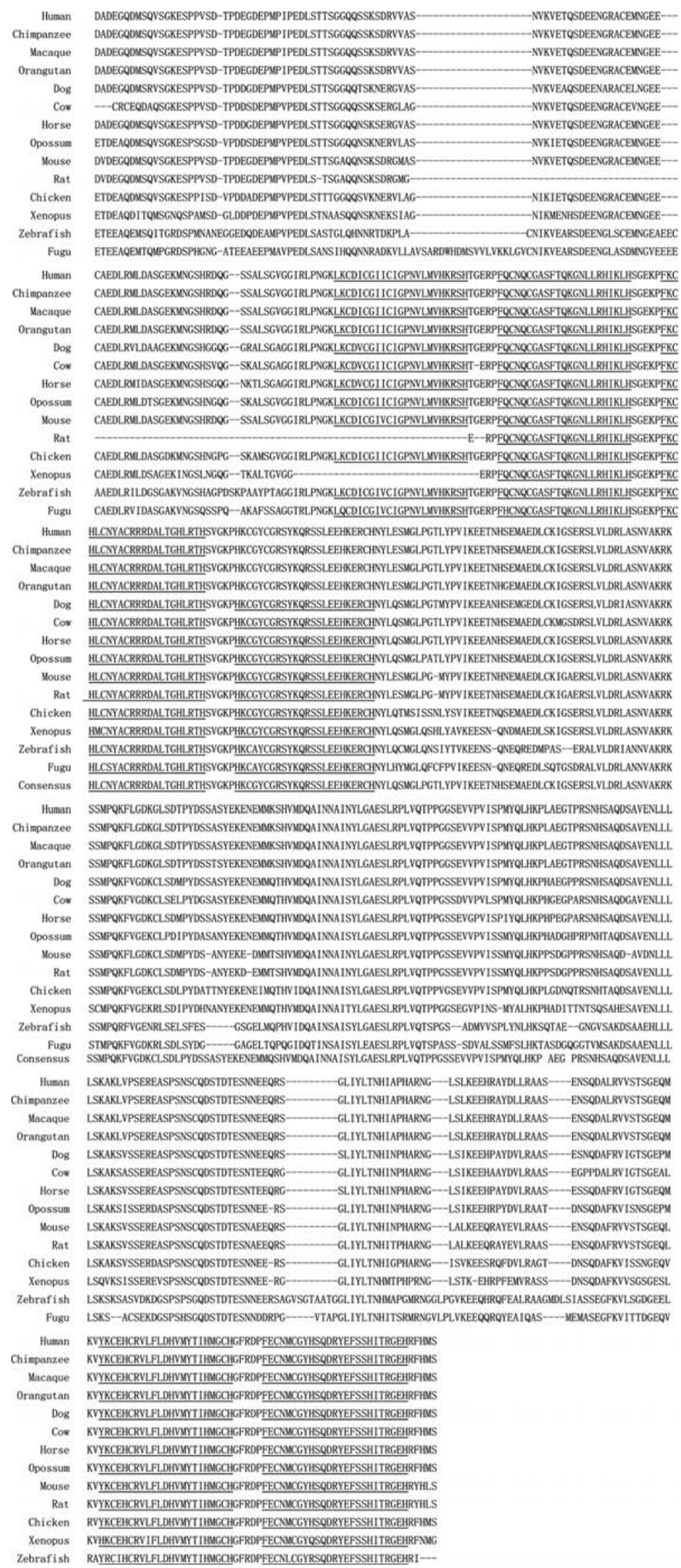

Figure 1. Alignments of amino acid sequences of identified Ikaros. Ikaros genes were identified in the genome sequences of human, chimpanzee, macaque, orangutan, dog, cow, horse, mouse, rat, opossum, chicken, Xenopus tropicalis, zebrafish, and fugu. Except rat and Xenopus tropicalis Ikaros proteins, which lack the first $\mathrm{C} 2 \mathrm{H} 2$-type 1 Zinc finger region, all identified Ikaros proteins containing six C2H2-type 1 Zinc finger regions. The C2H2-type 1 Zinc finger regions of identified Ikaros proteins are underlined. 


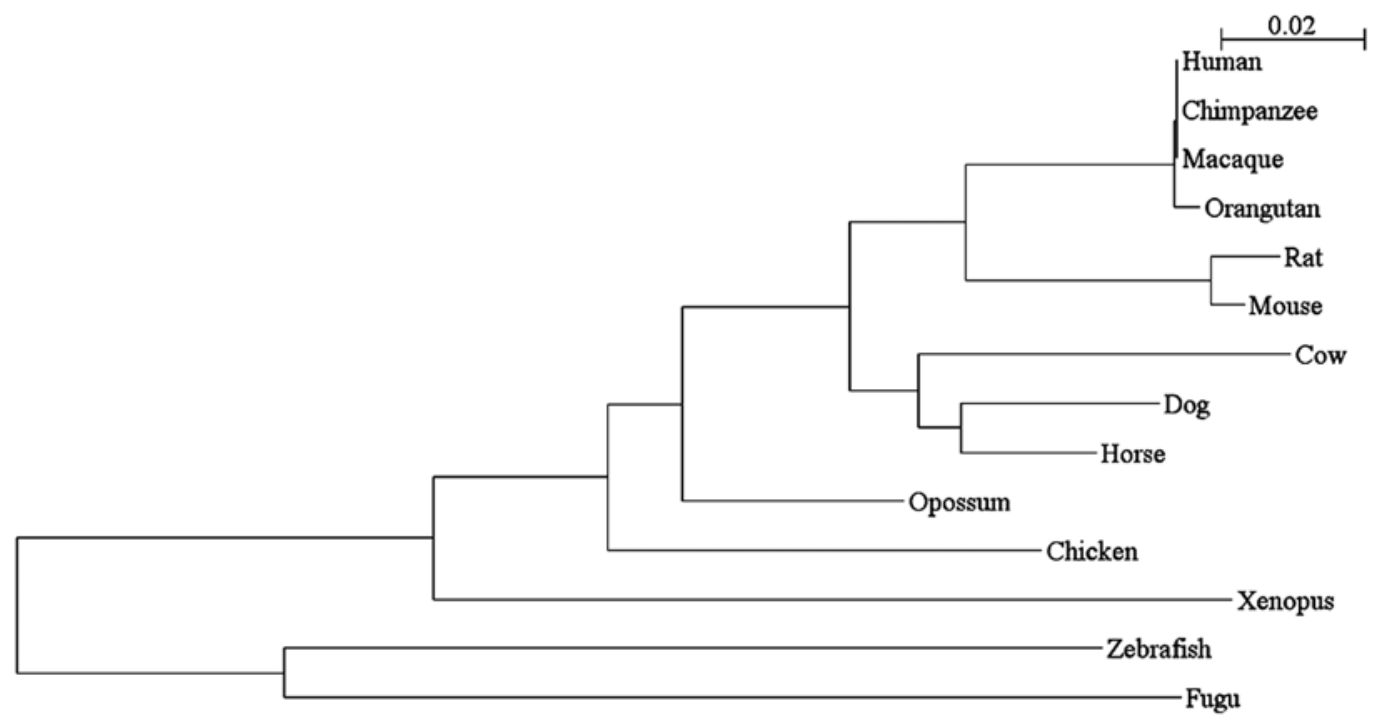

Figure 2. Phylogenetic analysis of Ikaros genes. The phylogenetic tree of Ikaros genes was obtained by using ML and NJ methods. The primate Ikaros clustered into one group, different from other Ikaros.

CTTCCGTGTGCTGGTGGATGCTTACTCTGTTTTCTGAAATACTTTTTCTGTACAGTGGCCACTAGCTGTACTCCTAAGCCACA CACCTACCTTGAAAATTCATGTCACTTTTAGAAATAGATAAAAGCCCCTCCCATCCAGAAAAAGTGACTATCATGTATATCCT CATCATGACTAATACTGATATTCCTGAAATTGAAAATACATATTCCATATGTACCATAAAAGGTATTAAAGATATATGGAGTG Elk-1

ATAGATATATTATATATAACACTTCTACCCTCACAGTTTTCAGCCTAATTGAGAGGGTAAGATCCCTGAATCATCCATCAGTT TTTCAGGTCTCTGCTGAAAGCAGGCCACAGCTCAGATCCACACATCTGAACCAGAGACAGAGGTGGCCAAAAATAAAAAGGGG GACAGGGGACAACCTGGTTTAGAGTCAACAAATAGACTGCATTTTCTGGTTAGTGAAGGAGCTCTCCTGAAAGTCATATACC AGAGCATAAATGAGCAGATTTCCTTGAGGTCACCTTCTGCTGGCCATAGCTTTCTTATCTGTGGAGCTGCCAGCTGTCATCCA CTTTGGGGCACCTGAGACTGCCGAGCGGCAGGCCAGGACCCAAGTGCGAAAACACAGAACACCTTTTTGTTTCTACTCCACTG ATGCTGGGGTTCTCTCCCTGGTGTTTGTGGCTCGTAGTACACTCTGTGGAACATTCACTATGGTCATCGAAGGGCAGCATCTT CCCAGTTGTTTCTTTCTTTTCTTTTTTTTTTTTAATTTAAACCGATCTGAGAAGCCAGCCATCTGTCAGCAAAACAGGAAGGC TCGGGCTGTCTCCTGGGCTCGTTTTGCTGCCGTAGTGAGCGTCACTTCTCCCCGTGTAAGAGTGCTGGTGAAGGCTGAGGCAA GGGCCCAGAAAGATTGAGGGACAAAGACAGGAGCGCCCGCATTGCCCATCTGCCAGGCTGGAGGTGTATTCATTATTGATGGA GGTAGTGCAGTTGCTGCTCAGATATGCAGCCCTGCCTGGGTAAATGAGACATTCTTCAGCAAATTGCTTCGTTTTTTGATTGC TGATTGTACGCGTGTCACCAAGCTGACTCAAGGTTCATCGATGCATGCTCAGTAAATTAGAAAGAACATAACTATGGATCAGC c-Fos

CAAGAGAATGAATTCTGTGCCTACAATGACCCAGGGCCATTTAATTTTCTGCTTAATTTTGTTGCAGTCAGTTTGCATTTTGG GTTATTATGCAGTAGGAAATTAACAATAAATAACAAATTTGGTCCTCCTGTGCTTGTAATGATATTTTTATAAATCTTTGTAA TGCTGTTTTTAAAAGGATCAAGGTCTGTGCCAGTCTGATACTCCAGCAAGTATGTGAGGAGGAAAATGCATTATTCTTGCTAG ATAACCTTGTT GTTAAATAGCATAGGGGTTCTTTATCTCTCTCTCTTTCTCATATCTTATTAGTATTTTTGCTTTAAACTAAA NKX6 GATA-1

ATCCCTTCCTCTCTTTCTCAGATAACCTGAGGACCATG

Figure 3. The identification of transcription factor-binding sites within the 5'-region of the human Ikaros gene. The transcription factor-binding sites are underlined. The transcriptional start sites (ATG) of human Ikaros is indicated in bold.

available SNPs were identified in human Ikaros gene. Among these, 5 SNPs were functionally relevant, including four available alleles disrupted an existing exonic splicing enhancer (rs11980379, rs11978363, rs6980115, rs11552047) and one SNP causing missense mutation (rs61731359). For rs61731359, an amino acid change from Asn to Asp in site 319 was reported. Moreover, two identical amino acid changes were also found for rs61731356 (site 391, Asn) and rs61731355 (site 333, Pro) (Table I).
Meta-analysis of the prognostic value of human Ikaros gene in cancer. When given the gene, PrognoScan displays a summary in table format of tests for the gene with columns for dataset, cancer type, subtype, endpoint, cohort, contributor, array type, probe ID, number of patient, optimal cut-point, Pmin and Pcor. Among the database detected expression of Ikaros, 14 out of 46 tests showed an association between microarray expression in Ikaros and cancer prognosis (bladder cancer $0 / 2$, blood cancer $4 / 9$, breast cancer $6 / 16$, 
Table I. Functional relevant SNP evaluation of the human Ikaros gene.

\begin{tabular}{llll}
\hline SNP ID & Chr 7 position & & Sequence \\
rs11980379 & $50437475(+)$ & GAAATTGTACATAAGT/CACCTCAGCATTTAAT & ESE \\
rs11978363 & $50437793(+)$ & TATTCCCAATATTCCC/TGGTCAGCAGTATCAA & ESE \\
rs6980115 & $50436016(+)$ & AGATTTTTATTTTTAG/CAGGCAGGGCTGCATT & ESE \\
rs11552047 & $50437045(+)$ & TCACCTGTTTGAAACC/TAAGCTTTCAAACATG & ESE \\
rs61731359 & $50435217(+)$ & ATCAACAACGCCATCA/GACTACCTGGGGGCC & Missense \\
rs61731356 & $50435435(+)$ & GGCGTCCCCGAGCAAC/TAGCTGCCAAGACTC & Synonymous \\
rs61731355 & $50435261(+)$ & GCTGGTGCAGACGCCA/CCCGGGCGGTTCCG & Synonymous \\
\hline
\end{tabular}

Four available alleles disrupted an existing exonic splicing enhancer (ESE), one SNP causing missense mutation (rs61731359), two SNPs (rs61731356 and rs61731355) causing two identical amino acid changes were identified.

Table II. Dataset content from PrognoScan showed an association between microarray expression in Ikaros and cancer prognosis.

\begin{tabular}{|c|c|c|c|c|c|c|c|c|}
\hline Database & $\begin{array}{l}\text { Case type } \\
\text { cancer }\end{array}$ & Subsyte & $\begin{array}{c}\text { Patients } \\
\text { no. }\end{array}$ & End-point & Cut-point & P-value & Prognosis & Refs. \\
\hline GSE12417-GPL96 & Blood & AML & 163 & Overall survival & 0.69 & 0.0209 & 1 & 42 \\
\hline E-TABM-346 & Blood & DLBCL & 53 & Event free survival & 0.34 & 0.0231 & 2 & 43 \\
\hline GSE16131-GPL97 & Blood & Follicular lymphoma & 180 & Overall survival & 0.22 & 0.0111 & 1 & 44 \\
\hline GSE2658 & Blood & Multiple myeloma & 559 & $\begin{array}{l}\text { Cause specific } \\
\text { survival }\end{array}$ & 0.31 & 0.0453 & 2 & 45 \\
\hline GSE6532-GPL570 & Breast & & 87 & $\begin{array}{l}\text { Recurrence } \\
\text { free survival }\end{array}$ & 0.13 & 0.0276 & 1 & 46 \\
\hline GSE9195 & Breast & & 77 & $\begin{array}{l}\text { Distant metastasis } \\
\text { free survival }\end{array}$ & 0.83 & 0.0297 & 1 & 47 \\
\hline GSE1379 & Breast & & 60 & $\begin{array}{l}\text { Recurrence } \\
\text { free survival }\end{array}$ & 0.47 & 0.0024 & 1 & 48 \\
\hline GSE2034 & Breast & & 286 & $\begin{array}{l}\text { Distant metastasis } \\
\text { free survival }\end{array}$ & 0.5 & 0.0138 & 1 & 49 \\
\hline E-TABM-158 & Breast & & 129 & $\begin{array}{l}\text { Distant metastasis } \\
\text { free survival }\end{array}$ & 0.74 & 0.0375 & 2 & 50 \\
\hline GSE3494-GPL96 & Breast & & 236 & $\begin{array}{l}\text { Disease specific } \\
\text { survival }\end{array}$ & 0.17 & 0.0016 & 1 & 51 \\
\hline GSE13213-2 & Lung & Adenocarcinoma & 30 & Overall survival & 0.87 & 0.0036 & 2 & 52 \\
\hline GSE4573 & Lung & $\begin{array}{l}\text { Squamous } \\
\text { cell carcinoma }\end{array}$ & 129 & Overall survival & 0.47 & 0.0237 & 1 & 53 \\
\hline DUKE-OC & Ovarian & & 134 & Overall survival & 0.64 & 0.0246 & 2 & 54 \\
\hline GSE19234 & Skin & Melanoma & 38 & Overall survival & 0.55 & 0.0268 & 1 & 55 \\
\hline
\end{tabular}

Fourteen tests showed an association between microarray expression in Ikaros and cancer prognosis (blood cancer 4/9, breast cancer $6 / 16$, lung cancer $2 / 10$, ovarian cancer $1 / 2$, skin cancer $1 / 1$ ) with $5 \%$ significance level. AML, acute myelocytic leukemia; DLBCL, diffuse large B-cell lymphoma; 1 represents poorer expression of Ikaros associated with poor survival; 2 represents higher expression of Ikaros associated with poor survival.

colorectal cancer $0 / 1$, gliomas $0 / 3$, head and neck cancer $0 / 1$, lung cancer $2 / 10$, ovarian cancer $1 / 2$, skin cancer $1 / 1$ ) with $5 \%$ significance level (Table II). Among the four blood cancers, we found a higher expression of Ikaros associated with poor survival in the case of diffuse large B-cell lymphoma (DLBCL) and multiple myeloma. However, a lower expression of Ikaros was related to poor survival in the case of diffuse large acute myeloid leukemia (AML) and 
follicular lymphoma. Among the six breast cancers, the higher expression of Ikaros was related to poor survival was found in only one case (E-TABM-158). Among the lung cases, we found a higher expression of Ikaros associated with poor survival in the case of adenocarcinoma and a lower expression of Ikaros associated with poor survival in the case of squamous cell carcinoma. Moreover, a higher expression of Ikaros associated with poor survival was found in the case of ovarian cancer, and a lower expression of Ikaros associated with poor survival in the case of skin cancer.

\section{Discussion}

Ikaros is a member of the Kruppel family of zinc finger DNA-binding proteins, which locates at 7 p12 in human genome. In the present study, we identified other Ikaros genes from other 13 vertebrate genomes and found Ikaros existed in all kinds of vertebrate including fish, amphibians, birds and mammals. Moreover, except rat and Xenopus tropicalis Ikaros proteins, which lack the first C2H2-type 1 Zinc finger region, all identified Ikaros proteins containing six C2H2-type 1 Zinc finger regions (Fig. 1). The phylogenetic tree shows that Ikaros is separated with the order fish, amphibians, birds and mammals, and primate Ikaros are almost the same and clustered together. From the alignment and phylogenetic tree, mammalian Ikaros are conversed among vertebrate genomes, suggesting that the function of Ikaros is essential for all the vertebrates in the long evolution process. Moreover, this process was under purifying selection.

Though Ikaros expression is essential for normal hematopoiesis in the lymphoid, myeloid, and erythroid lineages, it is not limited to the hematopoietic system. We found human Ikaros gene was expressed in many tissues and organs. It shows a predominant expression in the liver, lymph node, thymus, intestine, lung, mammary gland, bone marrow, brain, heart, placenta, and prostate. It implied Ikaros may be involved in the physiological functions of these tissues. Alternative splicing of Ikaros gene results in a number of mRNA and protein isoforms (Ik1-8) with distinct activity and capability of DNA binding regulating the role in controlling hematopoietic, particularly lymphoid cell differentiation, proliferation and function (3-6). Abnormalities in splicing regulation of Ikaros would lead to significant pathological manifestations $(3,4,8,9)$. We identified four available SNPs disrupting an existing exonic splicing enhancer, which may affect the alternative splicing of Ikaros. The effects of these SNPs on Ikaros physiological and pathological function need further investigation.

Ikaros is involved in apoptosis and cell cycle regulation in lymphocytes and thought as a hematological and pituitary tumor suppressor $(15,16)$. In the present study, we found Ikaros was widely expressed in solid tumors including bladder, blood, breast, colorectal cancer, gliomas, head and neck, lung, ovarian and skin cancer. Prognostic analysis of Ikaros has been reported only in AML (15). We also found a lower expression of Ikaros was related to poor survival in AML, this was confirmed by PrognoScan analysis. However, the PrognoScan analysis depicted statistical significance in other 13 tests (blood cancer 3/9, breast cancer $6 / 16$, lung cancer $2 / 10$, ovarian cancer $1 / 2$, skin cancer $1 / 1)$, which were not previously reported. It suggested that the expression of Ikaros was related to the prognosis of many cancers including hematological and solid cancers. The mechanism of Ikaros involved in the process of these tumors needed further investigation. It is important to note that relationship between the expression of Ikaros and prognosis varied in different cancers, even in the same cancer from different database. It implied that the function of Ikaros in these tumors may be multidimensional (Table II), not just as a tumor suppressor. The c-Fos, Elk-1, GATA-1 and NKX6-B binding sites were identified within the upstream of the transcriptional start site of human Ikaros gene. c-Fos is a cellular proto-oncogene belonging to the immediate early gene family of transcription factors. Members of the Fos family dimerise with C-Jun to form the AP-1 transcription factor, which has been implicated in transformation and progression of many cancers (39). ELK1, is a member of the Ets family of transcription factors, originally identified as a key regulator of immediate-early genes, such as FOS, which are rapidly and transiently induced following exposure to extracellular ligands that activate the MAP kinase pathways. ELK1 is also an oncogene implicated in transformation and progression of many cancers $(40,41)$. These two tumorrelated transcriptional factors (c-Fos and Elk-1) may be involved in the effect of Ikaros in tumors.

\section{Acknowledgements}

This project was sponsored by the National Natural Science Foundation of China (No. 30801016, 30972822).

\section{References}

1. Georgopoulos K, Moore DD and Derfler B: Ikaros, an early lymphoid-specific transcription factor and a putative mediator for $\mathrm{T}$ cell commitment. Science 258: 808-812, 1992.

2. Rebollo A and Schmitt C: Ikaros, Aiolos and Helios: transcription regulators and lymphoid malignancies. Immunol Cell Biol 81: 171-175, 2003.

3. Sun L, Heerema N, Crotty L, et al: Expression of dominantnegative and mutant isoforms of the antileukemic transcription factor Ikaros in infant acute lymphoblastic leukemia. Proc Natl Acad Sci USA 96: 680-685, 1999.

4. Olivero S, Maroc C, Beillard E, et al: Detection of different Ikaros isoforms in human leukaemias using real-time quantitative polymerase chain reaction. Br J Haematol 110: 826-830, 2000.

5. Payne KJ, Nicolas JH, Zhu JY, Barsky LW and Crooks GM: Cutting edge: predominant expression of a novel Ikaros isoform in normal human hemopoiesis. J Immunol 167: 1867-1870, 2001.

6. Payne KJ, Huang G, Sahakian E, et al: Ikaros isoform $\mathrm{x}$ is selectively expressed in myeloid differentiation. J Immunol 170: 3091-3098, 2003.

7. Cobb BS, Morales-Alcelay S, Kleiger G, Brown KE, Fisher AG and Smale ST: Targeting of Ikaros to pericentromeric heterochromatin by direct DNA binding. Genes Dev 14: 2146-2160, 2000 .

8. Nakayama H, Ishimaru F, Avitahl N, et al: Decreases in Ikaros activity correlate with blast crisis in patients with chronic myelogenous leukemia. Cancer Res 59: 3931-3934, 1999.

9. Tonnelle C, Calmels B, Maroc C, Gabert J and Chabannon C: Ikaros gene expression and leukemia. Leuk Lymphoma 43: 29-35, 2002.

10. Georgopoulos K, Bigby M, Wang JH, et al: The Ikaros gene is required for the development of all lymphoid lineages. Cell 79: 143-156, 1994.

11. Allman D, Sambandam A, Kim S, et al: Thymopoiesis independent of common lymphoid progenitors. Nat Immunol 4: 168-174, 2003. 
12. Mullighan CG, Goorha S, Radtke I, et al: Genome-wide analysis of genetic alterations in acute lymphoblastic leukaemia. Nature 446: 758-764, 2007

13. Mullighan CG, Miller CB, Radtke I, et al: BCR-ABL1 lymphoblastic leukaemia is characterized by the deletion of Ikaros. Nature 453: 110-114, 2008.

14. Mullighan CG, Su X, Zhang J, et al: Deletion of IKZF1 and prognosis in acute lymphoblastic leukemia. N Engl J Med 360: 470-480, 2009.

15. Meleshko AN, Movchan LV, Belevtsev MV and Savitskaja TV: Relative expression of different Ikaros isoforms in childhood acute leukemia. Blood Cells Mol Dis 41: 278-283, 2008.

16. Ezzat S, Yu S and Asa SL: Ikaros isoforms in human pituitary tumors: distinct localization, histone acetylation, and activation of the $5^{\prime}$ fibroblast growth factor receptor-4 promoter. Am J Pathol 163: 1177-1184, 2003.

17. Katoh Y and Katoh M: Integrative genomic analyses on GLI1: positive regulation of GLI1 by Hedgehog-GLI, TGFbeta-Smads, and RTK-PI3K-AKT signals, and negative regulation of GLI1 by Notch-CSL-HES/HEY, and GPCR-Gs-PKA signals. Int J Oncol 35: 187-192, 2009

18. Katoh Y and Katoh M: Integrative genomic analyses on GLI2: mechanism of Hedgehog priming through basal GLI2 expression, and interaction map of stem cell signaling network with P53. Int J Oncol 33: 881-886, 2008

19. Katoh Y and Katoh M: Comparative integromics on JMJD2A, JMJD2B and JMJD2C: preferential expression of JMJD2C in undifferentiated ES cells. Int J Mol Med 20: 269-273, 2007.

20. Katoh Y and Katoh M: Comparative genomics on PROM1 gene encoding stem cell marker CD133. Int J Mol Med 19: 967-970, 2007.

21. Katoh Y and Katoh M: Conserved POU-binding site linked to SP1-binding site within FZD5 promoter: transcriptional mechanisms of FZD5 in undifferentiated human ES cells, fetal liver/spleen, adult colon, pancreatic islet, and diffuse-type gastric cancer. Int J Oncol 30: 751-755, 2007.

22. Katoh $\mathbf{M}$ and Katoh $\mathrm{M}$ : Integrative genomic analyses on HES/ HEY family: Notch-independent HES1, HES3 transcription in undifferentiated ES cells, and Notch-dependent HES1, HES5, HEY1, HEY2, HEYL transcription in fetal tissues, adult tissues, or cancer. Int J Oncol 31: 461-466, 2007.

23. Katoh $\mathbf{M}$ and Katoh M: Comparative integromics on JMJD1C gene encoding histone demethylase: conserved POU5F1 binding site elucidating mechanism of JMJD1C expression in undifferentiated ES cells and diffuse-type gastric cancer. Int J Oncol 31: 219-223, 2007.

24. Katoh $\mathrm{M}$ and Katoh $\mathrm{M}$ : Integrative genomic analyses of WNT11: transcriptional mechanisms based on canonical WNT signals and GATA transcription factors signaling. Int $\mathrm{J} \mathrm{Mol}$ Med 24: 247-251, 2009.

25. Katoh $\mathrm{M}$ and Katoh $\mathrm{M}$ : Transcriptional mechanisms of WNT5A based on NF-kappaB, Hedgehog, TGFbeta, and Notch signaling cascades. Int J Mol Med 23: 763-769, 2009.

26. Katoh $\mathrm{M}$ and Katoh $\mathrm{M}$ : Integrative genomic analyses of ZEB2: transcriptional regulation of $\mathrm{ZEB} 2$ based on SMADs, ETS1, HIF1 alpha, POU/OCT, and NF-kappaB. Int J Oncol 34: 1737-1742, 2009.

27. Katoh $\mathrm{M}$ and Katoh M: Transcriptional regulation of WNT2B based on the balance of Hedgehog, Notch, BMP and WNT signals. Int J Oncol 34: 1411-1415, 2009

28. Yang L, Wei J and He S: Integrative genomic analyses on interferon $\lambda \mathrm{s}$ and their roles in cancer prediction. Int $\mathrm{J} \mathrm{Mol}$ Med 25: 299-304, 2010.

29. Thompson JD, Gibson TJ, Plewniak F, Jeanmougin F and Higgins DG: The CLUSTAL-X windows interface: flexible strategies for multiple sequence alignment aided by quality analysis tools. Nucleic Acids Res 15: 4876-4882, 1997.

30. Guindon S, Lethiec F, Duroux P and Gascuel O: PHYML Online, a web server for fast maximum likelihood-based phylogenetic inference. Nucleic Acids Res 33: W557-W559, 2005.

31. Kumar S, Tamura K and Nei M: MEGA3: integrated software for molecular evolutionary genetics analysis and sequence alignment. Brief Bioinform 5: 150-163, 2004

32. Yang Z: PAML: a program package for phylogenetic analysis by maximum likelihood. Comput Appl Biosci 13: 555-556, 1997

33. Yang Z, Nielsen R, Goldman N and Pedersen AM: Codonsubstitution models for heterogeneous selection pressure at amino acid sites. Genetics 155: 431-449, 2000.
34. Halama N, Grauling-Halama SA, Beder A and Jäger D: Comparative integromics on the breast cancer-associated gene KIAA1632: clues to a cancer antigen domain. Int J Oncol 31: 205-210, 2007.

35. Halama N, Grauling-Halama SA and Jäger D: Identification and characterization of the human StARD9 gene in the LGMD2Aregion on chromosome $15 \mathrm{q} 15$ by in silico methods. Int $\mathrm{J}$ Mol Med 18: 653-656, 2006.

36. Chalifa-Caspi V, Yanai I, Ophir R, et al: GeneAnnot: comprehensive two-way linking between oligonucleotide array probesets and GeneCards genes. Bioinformatics 20: 1457-1458, 2004.

37. Parkinson H, Sarkans U, Shojatalab M, et al: ArrayExpress, a public repository for microarray gene expression data at the EBI. Nucleic Acids Res 33 (Database issue): D553-D555, 2005.

38. Mizuno H, Kitada K, Nakai K and Sarai A: PrognoScan: a new database for meta-analysis of the prognostic value of genes. BMC Med Genomics 2: 18, 2009.

39. Durchdewald M, Angel $P$ and Hess J: The transcription factor Fos: a Janus-type regulator in health and disease. Histol Histopathol 24: 1451-1461, 2009.

40. Boros J, Donaldson IJ, O'Donnell A, et al: Elucidation of the ELK1 target gene network reveals a role in the coordinate regulation of core components of the gene regulation machinery. Genome Res 11: 1963-1973, 2009.

41. Rostad K, Mannelqvist M, Halvorsen OJ, et al: ERG upregulation and related ETS transcription factors in prostate cancer. Int J Oncol 30: 19-32, 2007.

42. Metzeler KH, Hummel M, Bloomfield CD, et al: An 86probe-set gene-expression signature predicts survival in cytogenetically normal acute myeloid leukemia. Blood 112: 4193-4201, 2008.

43. Jais JP, Haioun C, Molina TJ, et al: The expression of 16 genes related to the cell of origin and immune response predicts survival in elderly patients with diffuse large B-cell lymphoma treated with CHOP and rituximab. Leukemia 22: 1917-1924, 2008.

44. Leich E, Salaverria I, Bea S, et al: Follicular lymphomas with and without translocation $\mathrm{t}(14 ; 18)$ differ in gene expression profiles and genetic alterations. Blood 114: 826-834, 2009.

45. Zhan F, Huang Y, Colla S, et al: The molecular classification of multiple myeloma. Blood 108: 2020-2028, 2006.

46. Loi S, Haibe-Kains B, Desmedt C, et al: Definition of clinically distinct molecular subtypes in estrogen receptor-positive breast carcinomas through genomic grade. J Clin Oncol 25: 1239-1246, 2007.

47. Loi S, Haibe-Kains B, Desmedt C, et al: Predicting prognosis using molecular profiling in estrogen receptor-positive breast cancer treated with tamoxifen. BMC Genomics 9: 239, 2008.

48. Ma XJ, Wang Z, Ryan PD, et al: A two-gene expression ratio predicts clinical outcome in breast cancer patients treated with tamoxifen. Cancer Cell 5: 607-616, 2004.

49. Wang Y, Klijn JG, Zhang Y, et al: Gene-expression profiles to predict distant metastasis of lymph-node-negative primary breast cancer. Lancet 365: 671-679, 2005.

50. Chin SF, Wang Y, Thorne NP, et al: Using array-comparative genomic hybridization to define molecular portraits of primary breast cancers. Oncogene 26: 1959-1970, 2007.

51. Miller LD, Smeds J, George J, et al: An expression signature for p53 status in human breast cancer predicts mutation status, transcriptional effects, and patient survival. Proc Natl Acad Sci USA 102: 13550-13555, 2005

52. Tomida S, Takeuchi T, Shimada Y, et al: Relapse-related molecular signature in lung adenocarcinomas identifies patients with dismal prognosis. J Clin Oncol 27: 2793-2799, 2009.

53. Raponi M, Zhang Y, Yu J, et al: Gene expression signatures for predicting prognosis of squamous cell and adenocarcinomas of the lung. Cancer Res 66: 7466-7472, 2006.

54. Bild AH, Yao G, Chang JT, et al: Oncogenic pathway signatures in human cancers as a guide to targeted therapies. Nature 439 : 353-357, 2006

55. Bogunovic D, O'Neill DW, Belitskaya-Levy I, et al: Immune profile and mitotic index of metastatic melanoma lesions enhance clinical staging in predicting patient survival. Proc Natl Acad Sci USA 106: 20429-20434, 2009. 